\title{
The deep sulcus sign indicates free air in the abdomen
}

\author{
Sheng-Huei Wang ${ }^{2} \cdot$ Kuang-Yu Wei ${ }^{2} \cdot{\text { Yao-Chia } \text { Liu }^{3} \cdot \text { Chao-Hsin Huang }}^{1}$
}

Received: 10 May 2015/Accepted: 15 May 2015/Published online: 28 May 2015

(C) SIMI 2015

An 83-year-old man was admitted to our intensive care unit after endotracheal intubation for pneumonia associated with acute respiratory failure. He had a history of chronic obstructive pulmonary disease controlled with medication. Dyspnea and oxygen desaturation developed on day 7. A follow-up supine chest radiograph showed a linear radiolucent area in the right upper abdominal quadrant (Fig. 1a). Physical examination revealed mild upper abdominal tenderness and bilateral chest crackles. Computed tomography indicated a right pneumothorax and generalized emphysematous change of lung tissue (Fig. 1b). After placement of a chest tube, the clinical manifestations im- proved, and the radiolucent lesion disappeared on the subsequent chest radiograph (Fig. 1c).

The deep sulcus sign, indicating a pneumothorax, appears as a continuous, radiolucent, and deep costophrenic angle on a supine chest radiograph. Intrapleural air distributes in a non-dependent way, and accumulates from the anteromedial region to the laterocaudal region as the pneumothorax increases [1]. In our case, the pneumothorax may have resulted from rupture of a bulla in the right upper lung. A pleural line due to expansion of the right posterior lobe of the lung was not visible on chest radiography. The deep sulcus sign was interrupted by adhesion of lung tissue

Chao-Hsin Huang

slider4520@icloud.com

1 Division of Pulmonary Medicine, Department of Internal Medicine, Tri-service General Hospital Songshan Branch, Taipei, Taiwan, ROC

2 Department of Internal Medicine, Tri-Service General Hospital, National Defense Medical Center, Taipei, Taiwan, ROC

3 Department of Anesthesiology, Tri-Service General Hospital, National Defense Medical Center, Taipei, Taiwan, ROC 
Fig. 1 a Chest radiograph showing a linear radiolucent area (arrow) in the right upper abdominal quadrant. c After thoracostomy, the radiolucent lesion on chest radiograph disappeared. Computed tomographic scans showing b the right pneumothorax and a generalized emphysematous change of lung tissue and c adhesion (arrow) of lung tissue to the parietal pleura, interrupting the deep sulcus sign

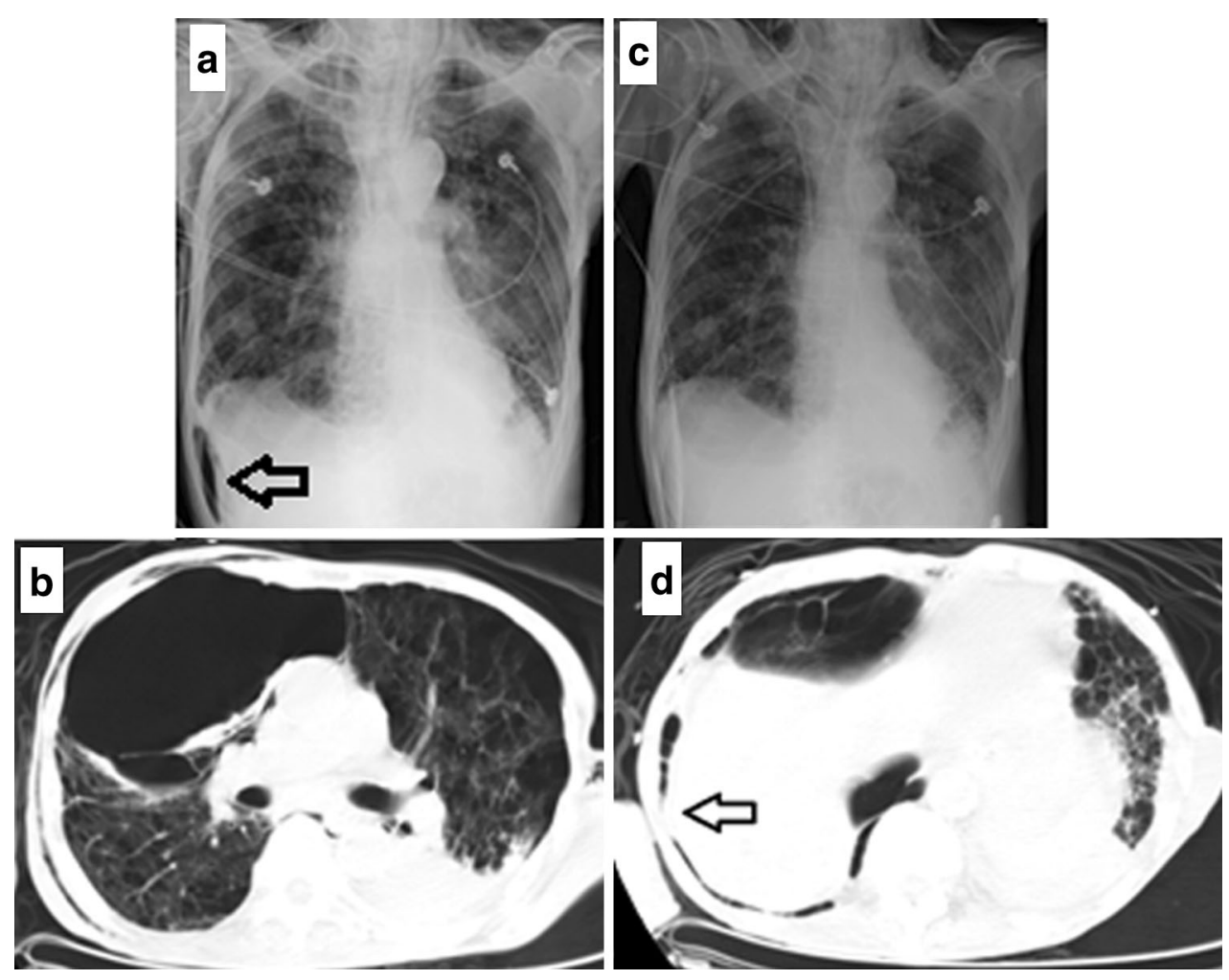

to the parietal pleura, which may explain the resemblance to free air in the abdomen (Fig. 1d). Early diagnosis of a pneumothorax based on the deep sulcus sign is crucial in clinical practice.

Conflict of interest The authors declare they have no conflict of interest.

Statement of human and animal rights All procedures performed in studies involving human participates were in accordance with the ethical standards of the national research committee and with the 1964 Helsinki declaration and comparable ethical standards. This article does not contain any studies with animals performed by any of the author.

Informed consent Informed consent was obtained from all individual participates included in this study.

\section{Reference}

1. Sabbar S, Nilles EJ (2012) Deep sulcus sign. N Engl J Med 366:552 